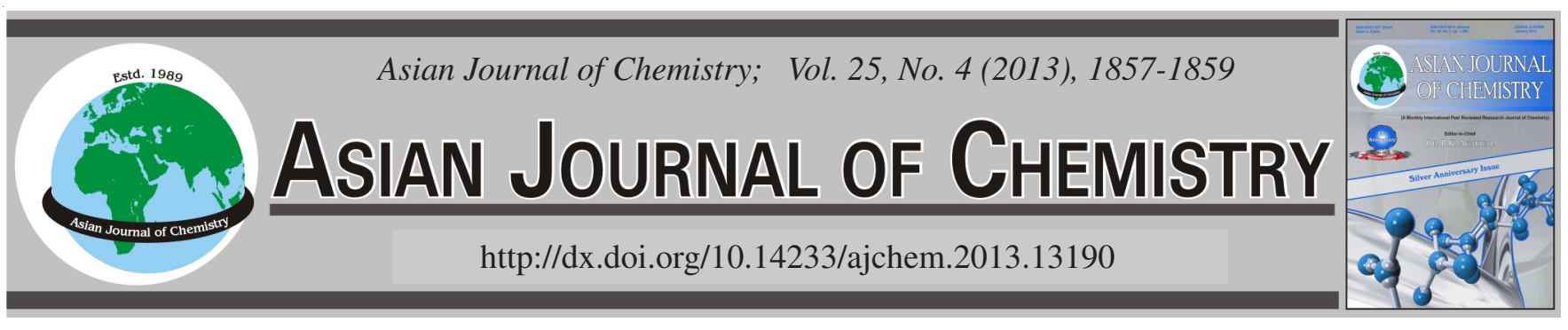

\title{
Study of Chromatographic Column Packing and Its Application
}

\author{
Z.Q. LIU*, R.C. LiU and F.Y. JIANG
}

School of Environmental and Material Engineering, Yantai University, Yantai, P.R. China

*Corresponding author: Fax: +86 535 6706038; Tel: +86 535 6706038; E-mail: lzqytu@ 163.com

This experiment used analyzer and chromatography to make the evaluation of chromatographic packing materials based on silica by means of analysis, separation and purification. Reverse phase silica was prepared by octadecyltrichlorosilane $\left(\mathrm{C}_{18}\right)$ under normal lab conditions. The column was filled with the prepared silica as chromatographic packing materials and the column efficiency was also evaluated. Then lignans was isolated of from natural extracts using the prepared column. The lignans was purified with high pressure and its purity was also tested with HPLC. In addition the procedure was repeated many times so that the article discussed and tested the performance of chromatographic packing materials based on silica.

Key Words: Silica, Chromatography, Chromatographic packing materials.

ᄂ - - - - - - - - - - - - - - -

\section{INTRODUCTION}

Inorganic packing is the mainstream in the research and application of high-performance liquid chromatographic packing. Among all of inorganic packing, the research and application of silica-based packing is essential. And in bonded phase chromatographic packing which uses silica gels and silica based as, reverse phase bonded silica is most commonly used. Further among these kinds of reverse phase bonded silica, octadecyltrichlorosilane $\left(\mathrm{C}_{18}\right)$ accounts for over $90 \%$. It is the appearance of silica particles that fosters the appearance of high-performance liquid chromatograms. With the development of the performance of silica gels, high-performance liquid chromatograms are widely used ${ }^{1,2}$.

Silica and bonded phase silica are the HPLC stationary phase that is developed earliest, extensively researched and widely used. This is mainly based on the good physical and chemical features of silica-body. We can control the manufacture craft of porous silica particles to make packing that have a wider average aperture alteration, a narrower aperture scatter and more options of granularity. It can meet the demand of the analysis and making of big and small molecules. The outstanding merit of most silica particles is that they have a good mechanical strength and it can ensure the steadiness of column efficiency when the packed beds work under great operation forces for a long time. Under rigid and high intensity condition, the prepared particles also make column has a lower counter pressure and a longer life. It is also an important merit of the silica-based column that the column efficiency of it is the highest among materials that are used to make packing ${ }^{3-6}$. Generally lab-made silica stationary phase could not be comparable with the plant mass ones. However, under suitable conditions, bonded silica stationary phase made in the lab has high column efficiency relatively. Present experiment uses $\mathrm{C}_{18}$ to make reverse silica under normal lab conditions and use the prepared column to evaluate the column efficiency. Then we use silica to make the chromatographic columns to extract lignanoid from natural extracts. We also use high-pressure equipments to purify lignanoid and HPLC analysis to track the lignanoid and measure the purity. The procedure is repeated many times, thus we can evaluate the performance of silica bonded packing that is made. Present experiment connects high-performance liquid chromatograms and silica gel, the inorganic materials. We learn from it that the application of material in the chromatograms boosts the development of chromatographic technology and the technology in turn provides wider space for the application of the material.

\section{EXPERIMENTAL}

Shimadzu High-performance Liquid Chromatographic Instrument (LC-10ATvp); Elite high-performance liquid chromatographic instrument (UV230+-P270); Rotary evaporator (R201D); electronic balance (JA1003); high and medium pressure column $(36 \times 460 \times 26 \times 310)$; Ultrasonic cleaner (EQ22OODE).

Chromatographic column filling material SinoChrom ODS-AP120A20-50 $\mu \mathrm{m}$ (Dalian elite analytical instruments 
Co. Ltd.), methanol (HPLC grade) (Product of Tedia, USA); Acetonitrile (HPLC grade) (Product of Tedia, USA); ammonium formate (AR); toluene (AR); naphthalene (AR); trifluoroacetic acid (TFA) (AR); N,N-dimethylformamide (DMF) (AR).

\section{General procedure}

Using $\mathbf{C}_{18}$ to make reverse phase silica: Take $50 \mathrm{~g} 50 \mu \mathrm{m}$ silica gel to let it go through cleanup acid treatment by 500 $\mathrm{mL} 10 \% \mathrm{HCl}$ in the three-neck flask with reflux condensing tube and propeller-type agitator. In the oil bath we heat hydrochloric acid to reflux and adjust the stirring speed to make the silica suspended during heating process. This procedure may take 6-8 h. After the process, leach the liquid with sand core funnel, wash out with distilled water is slightly acidic to neutral solution ( $\mathrm{pH}$ 5.5-7.0), then place in oven at $100-110^{\circ} \mathrm{C}$ drying. And we make reverse phase silica particles with the grain diameter of $30 \mu \mathrm{m}$ using the same method.

Choice of making glass tubes of chromatographic column: The material of making glass tubes of chromatographic column is borosilicate glass. The main components of the glass are $\mathrm{SiO}_{2}, \mathrm{CaO}, \mathrm{Na}_{2} \mathrm{O}, \mathrm{Al}_{2} \mathrm{O}_{3}, \mathrm{~B}_{2} \mathrm{O}_{3}$ and some other oxides, of which $\mathrm{SiO}_{2}$ is the most, accounting for over $80 \%$. Although there are not many oxides, still they have a large effect on the performance of glass. The adding of such components like $\mathrm{CaO}, \mathrm{Na}_{2} \mathrm{O}$ and $\mathrm{B}_{2} \mathrm{O}_{3}$ can break $\mathrm{Si}-\mathrm{O}-\mathrm{Si}$ in the structure of the glass, so that the melting point of such glass is lower than that of the quartz glass and is $c a$. 800$825^{\circ} \mathrm{C}$. In addition, as this kind of glass contains $\mathrm{B}_{2} \mathrm{O}_{3}$, it is acidic, strong and hard to break.

Filling in the making of chromatographic columns: The experiment adopts the wet method to fill as it has higher column efficiency. We pour the reverse phase silica that has been made into the $1000 \mathrm{~mL}$ beaker, add $500 \mathrm{~mL}$ methanol, stir it with the glass rod and pour it into the intensified glass tube which is sealed at the bottom. Then wait for it to subside naturally. When the lower layer subsides completely, use the injector to siphon out the methane of the upper layer and continue pouring reverse phase silica. The process is repeated for a few times until the glass tube is filled with the silica packing. Take down the column packing device, seal it at the top. Then the filling is completed.

Making of the sample: The procedures of the experiment: After adding $3 \mathrm{~mL}$ DMF for $1 \mathrm{~g}$ sample, dissolve it with $10 \mathrm{~mL}$ methanol in ultrasonic device and get the original sample. We analyze the sample and determine the appearance time and per cent of the impurity to be extracted. Then we extract the original sample (i.e., the enrichment of the sighting vane), analyze the latter sample and repeat the action until adequate sighting vanes are extracted roughly. We use the rotator evaporator to remove $30 \%$ of the methane, remove the water and remove the methane with the machine. Then make the packing under high pressure. At last analysis that after high-pressure making.

\section{RESULTS AND DISCUSSION}

The resulted data should be well presented by the form of schemes, figures, graphs, tables, reactions and equations. These items should be numbered clearly.
We choose to use medium pressure column of $36 \mathrm{~mm} \times$ $460 \mathrm{~mm} \times 30 \mu \mathrm{m} \mathrm{C}_{18}$ to extract roughly for the making for many times. We can figure out from the Fig. 1 that the sample has a good repetitiveness.
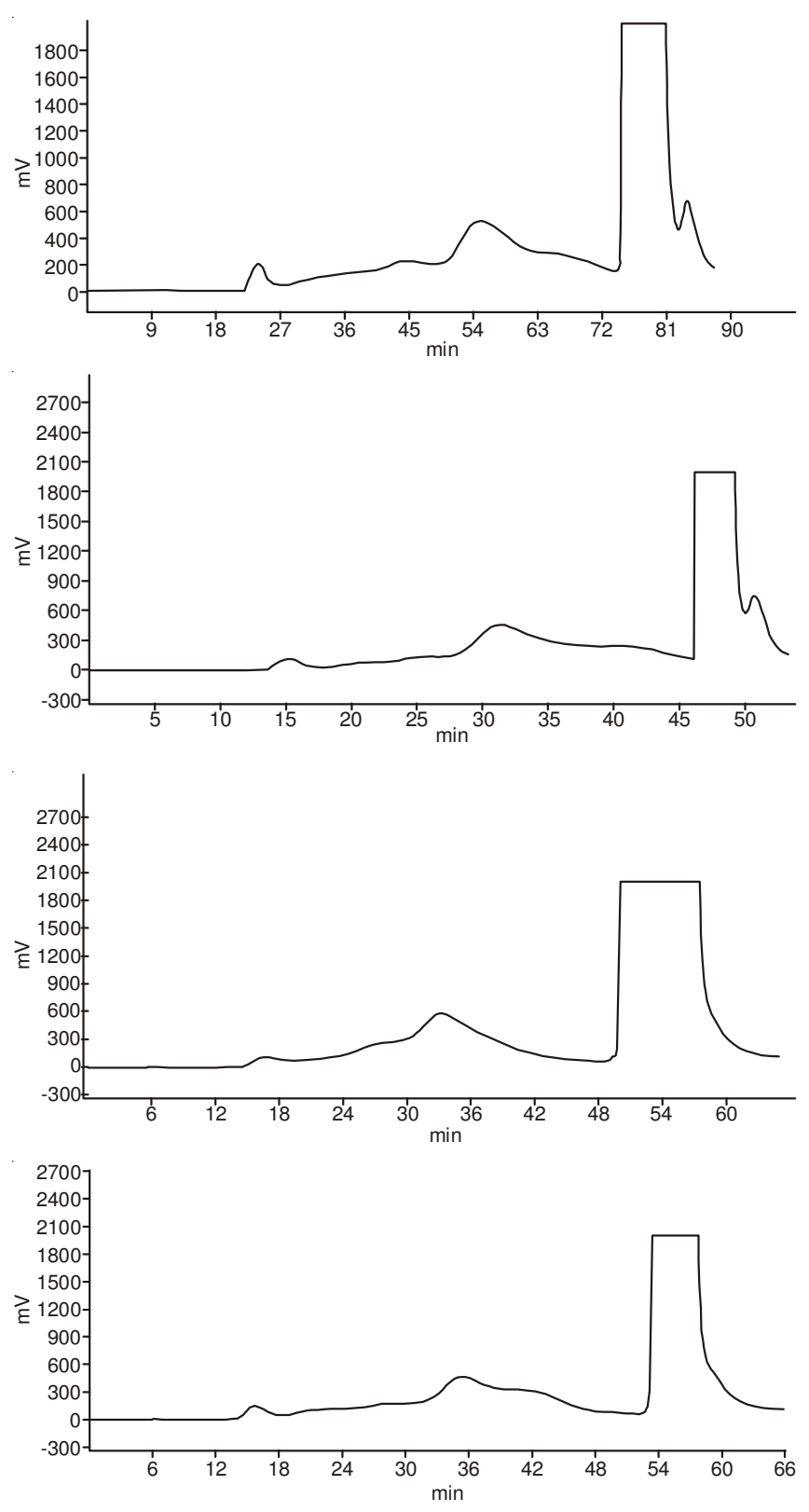

Fig. 1. Many times for rough preparation diagram

The filling material to make chromatogram in this experiment, that is reverse phase silica of bonded $\mathrm{C}_{18}$, is around 30 and $50 \mu \mathrm{m}$. It is much bigger than chromatographic analysis of filler (usually between 5-15 $\mu \mathrm{m}$ ). So the peak shape of preparation spectra is short and stout, it has a low degree of symmetry, a small separating size and can be preserved for a relatively long time, while the peak pattern of analysis spectra is high and sharp, it has a high degree of symmetry, a big separating size and can be preserved for a relatively short time. The silica gel has certain advantaged because it is big: it costs less, has a relatively simple manufacturing technique, the chromatographic has a lower pressure, fast flowing speed and larger sample size. 
Normally preparation column packing which is around 30 and $50 \mu \mathrm{m}$ can be able to extract and purify the required components from the biochemistry samples, natural mixtures and some other mixtures. Once we find a suitable method, we can increase the purity to be over $99 \%$ with solely medium pressure preparation.

The condition not being changed, spectra of rough preparation for many times should be same. If the repetitiveness is not good, it signifies that there is something wrong with certain parts.

The preparation spectra and analysis ones compared, it should be consistent, that is contained in the preparation of components in the analysis should also be there, otherwise the errors.

The polarity of the solution has a great influence on the analysis, especially when the material to be extracted has acidic or alkaline radicals or even has both.

\section{Conclusion}

Our experiment connected silica gel in the inorganic nonmetal materials and high-performance liquid chromatogram in analytical chemistry. We synthesized column stationary of reverse phase by binding $\mathrm{C}_{18}$ on the surface of commercial silica particles. Also we used dry and wet methods to pack preparative columns of different sizes separately. Then we used the columns to extract and purify natural extracts, medicine and so on. And the prepared silica packing was also test. Three conclusions were arrived as follows: (i) chemical bonding silica stationary phase is obviously superior in the application of high-performance liquid chromatogram. It reduces point mount of surface-active role to a large extent and eliminates certain possible catalytic activity. It is hard to be flushed by solvent. It has a good thermal stability. It is flexible in surface modification and can easily get repeated products. (ii) the smaller the silica particle is, the more even the packing is. Also the column efficiency is higher and (iii) as the size of particles is too tiny to be packed evenly, the most important thing for us to do is to choose a suitable filling methods.

At last, we need to enrich first when extract things that have a small amount. The process of enrichment can be operated in different periods.

\section{ACKNOWLEDGEMENTS}

This work was supported by National Natural Science Foundation of China (50972123) and Promotive research fund for Excellent Young and Middle-Aged Scientists of Shandong Province (2008BS05006).

\section{REFERENCES}

1. Q.H. Zhang, L.J. Yan and W.B. Zhang and Y.K. Zhang, Chin. J. Anal. Chem., 36, 572 (2008).

2. H.F. Zou, Z. Liu and M.L. Ye, The Capillary Electrochromatography and Application, Science Press, Beijing, p. 30 (2001).

3. L.J. Yan, Q.H. Zhang and Y.Q. Feng, W.-B. Zhang, T. Li, L.-H. Zhang and Y.-K. Zhang, J. Chromatogr. A, 1121, 92 (2006).

4. L.J. Yan, Q.H. Zhang and W.B. Zhang, Y.Q. Feng, L.H. Zhang, T. Li and Y.K. Zhang, Electrophroesis, 26, 2935 (2005).

5. H.L. Luo, J. Sheng and Y.Z. Wan, Mater. Lett., 62, 37 (2008).

6. T.J. Yao, Q. Lin, K. Zhang, D.F. Zhao, H. Lv, J.H. Zhang and B. Yang, J. Colloid Interf. Sci., 315, 434 (2007). 